\title{
BMJ Open Is there a causal relationship between acute stage sensorimotor cortex activity and the development of chronic low back pain? a protocol and statistical analysis plan
}

\author{
Luke Jenkins, ${ }^{1,2}$ Wei-ju Chang, ${ }^{1,2}$ Valentina Buscemi, ${ }^{1,2}$ Chelsea Cunningham, ${ }^{2}$ \\ Aidan Cashin, ${ }^{2,3}$ James H McAuley, ${ }^{2,4}$ Matthew Liston, ${ }^{1,2}$ Siobhan M Schabrun (D) ${ }^{2}$
}

To cite: Jenkins L, Chang W, Buscemi $\mathrm{V}$, et al. Is there a causal relationship between acute stage sensorimotor cortex activity and the development of chronic low back pain? a protocol and statistical analysis plan. BMJ Open 2019;9:e035792. doi:10.1136/ bmjopen-2019-035792

- Prepublication history for this paper is available online. To view these files, please visit the journal online (http://dx.doi. org/10.1136/bmjopen-2019035792).

Received 18 November 2019 Revised 27 November 2019 Accepted 28 November 2019

Check for updates

(C) Author(s) (or their employer(s)) 2019. Re-use permitted under CC BY-NC. No commercial re-use. See rights and permissions. Published by BMJ.

For numbered affiliations see end of article.

Correspondence to Dr Siobhan M Schabrun; s.schabrun@neura.edu.au

\section{ABSTRACT}

Introduction Why some people develop chronic pain following an acute episode of low back pain is unknown. Recent cross-sectional studies have suggested a relationship between aberrant sensorimotor cortex activity and pain persistence. The UPWaRD (Understanding persistent Pain Where it ResiDes) cohort study is the first prospective, longitudinal investigation of sensorimotor cortex activity in low back pain. This paper describes the development of a causal model and statistical analysis plan for investigating the causal effect of sensorimotor cortex activity on the development of chronic low back pain.

Methods and analysis Sensorimotor cortex activity was assessed within 6 weeks of low back pain onset using somatosensory evoked potentials and transcranial magnetic stimulation mapping techniques. Chronic low back pain is defined as ongoing pain (Numerical Rating score $\geq 1$ ) or disability (Roland Morris Disability Questionnaire score $\geq 3$ ) at 6 months follow-up. Variables that could confound the relationship between sensorimotor cortex activity and chronic low back pain were identified using a directed acyclic graph and content expertise was used to specify known causal paths. The statistical model was developed 'a priori' to control for confounding variables identified in the directed acyclic graph, allowing an unbiased estimate of the causal effect of sensorimotor activity in acute low back pain on the development of chronic pain. The statistical analysis plan was finalised prior to follow-up of all participants and initiation of analysis. Ethics and dissemination Ethical approval has been obtained from Western Sydney University Human Research Ethics Committee (H10465) and from Neuroscience Research Australia (SSA: 16/002). Dissemination will occur through presentations at national and international conferences and publications in international peerreviewed journals.

Trial registration number ACTRN12619000002189 (retrospectively registered)

\section{BACKGROUND}

Low back pain (LBP) is the most common form of persistent musculoskeletal pain and a
Strengths and limitations of this study

- The causal objective for data obtained from the UPWaRD (Understanding persistent Pain Where it ResiDes) study is made explicit and transparent within this protocol and analysis plan.

- Acknowledging the causal goal of this research can inform scientific discussion of future results.

- Detailed description of confounder selection using a directed acyclic graph is transparently reported 'a-priori'.

- A causal analysis in observational data can be viewed as an attempt to emulate a hypothetical trial — 'the target trial'. Currently, it remains challenging to sufficiently define sensorimotor cortex activity as an 'intervention'.

- There can be no guarantee that a causal model incorporates all confounders.

leading cause of disability. ${ }^{1}$ In 2012, the direct healthcare costs of LBP in Australia were estimated at $\$ 4.8$ billion, ${ }^{2}$ while in the USA, this figure approaches $\$ 50$ billion. ${ }^{3}$ Most of these costs are associated with chronic LBP, that is, pain that has persisted for more than 3 months. It is not understood why up to $40 \%$ of people with acute LBP develop chronic LBP. $^{4}$ Interventions to prevent the development of chronic LBP have not been effective. ${ }^{5}$ Identifying the causal mechanisms that explain why some people develop chronic LBP may guide the development of targeted treatment and is considered a research priority. ${ }^{67}$

Causes of a health condition are defined as characteristics or events necessary for the condition to occur, ${ }^{8}$ that is, "had the exposure differed, the outcome would have differed'. ${ }^{\prime}$ Aberrant sensorimotor cortex activity in the acute stage of LBP is one characteristic 
postulated to have a causal relationship with the development of chronic pain. ${ }^{10-12}$ Cross-sectional studies have shown larger activity and a shift in the S1 representation of the back ${ }^{11}$ and enlarged, overlapped and shifted M1 representations of the back muscles in chronic LBP compared with pain-free individuals, ${ }^{13}{ }^{14}$ and these changes are associated with pain, functional impairment and symptom chronicity. ${ }^{14-18}$ Further, preliminary evidence suggests sensorimotor cortex activity in acute LBP is lower in patients with acute clinical LBP than in pain-free controls. ${ }^{10}$ Despite these findings, no study has investigated the causal relationship between sensorimotor cortex activity in acute LBP and the development of chronic pain.

Recent conceptual advances have outlined methods for estimating the causal effect of an exposure on a health outcome using observational data. ${ }^{19}$ Two major considerations when attempting to estimate causal effect include how a particular target trial is emulated with observational data and the appropriate selection of confounding variables. ${ }^{20}{ }^{21}$ Identification and inclusion of confounding variables in a statistical model is essential to estimate causal effects. ${ }^{21}$ Confounding occurs when an exposure and outcome share a common cause. ${ }^{22-24}$ Data driven identification of confounding variables such as $p$ value based and model-based variable selection methods ignore the causal structure underlying the hypothesis and subsequently do not aid in causal inference. ${ }^{15} 18$ Rather, expert knowledge is required to specify the causal structure. $^{25}$ Causal models can be represented visually using directed acyclic graphs (DAGs). ${ }^{9}{ }^{19-29}$ A DAG provides a graphical representation of a mathematically rigorous method for minimising confounding bias within observational research. ${ }^{89}$ While there can be no certainty a causal model incorporates all known confounders, this approach to identifying confounding bias and developing a causal model makes assumptions explicit and transparent, promoting informed scientific discussion. ${ }^{20}$

Using data from the UPWaRD (Understanding persistent Pain Where it ResiDes) prospective, longitudinal cohort study, this paper reports the development of a causal model to investigate whether sensorimotor cortex activity (exposure) in the acute stage of LBP has a causal effect on the development of chronic LBP (outcome). First we describe the protocol for data collection and development of a DAG, detailing the explicit assumptions for identification of confounding variables within a causal model of chronic LBP. ${ }^{19}$ Second, we report a prespecified statistical analysis plan in line with the STROBE (Strengthening the Reporting of Observational Studies in Epidemiology) statement. ${ }^{30}$

\section{METHODS}

\section{Design}

The UPWaRD study is a multicentre, prospective, longitudinal, cohort study of people presenting with acute LBP (National Health and Medical Research Council of
Australia, Grant ID: 1059116). The study was registered with the Australian and New Zealand Clinical Trials Registry and the full study protocol has been published. ${ }^{31}$ The study enrolled 120 participants with acute LBP, with each participant undergoing a battery of neurophysiological and psychological tests at baseline with follow-up completed at 6 months.

\section{Patient and public involvement}

Patients and the public were not involved in the design of this protocol and statistical analysis plan. Patient advocacy groups (Chronic Pain Australia, Pain Australia) provided support for recruitment through dissemination of recruitment flyers in newsletters, websites and social media. Individual test results will be provided to participants on request and a summary of the overall outcomes of the study will be available to all participants on completion of the trial.

\section{Objective}

The primary aim of the UPWaRD study was to determine whether sensorimotor cortex activity, an individual's capacity for neuroplasticity, and psychosocial features, assessed during an acute episode of LBP could predict 6 month LBP outcome. ${ }^{31}$ As predictive models have different aims to studies investigating causal inference, this paper outlines the statistical analysis plan for using data obtained from the UPWaRD study to investigate whether sensorimotor cortex activity has a causal effect in the development of chronic LBP.

\section{Hypothesis}

Null hypothesis: Sensorimotor cortex activity in the acute stage of LBP (T1) does not cause chronic LBP at 6 months. The null hypothesis will be rejected if sensorimotor cortex activity demonstrates a significant causal relationship with chronic LBP (pain or disability) at 6 month follow-up (T2).

\section{Inclusion criteria}

Participants were eligible for inclusion in the study if the following criteria were met:

- 18 years or older and experiencing acute non-specific LBP - defined as pain in the region of the lower back, superiorly bound by the thoracolumbar junction and inferiorly by the gluteal fold ${ }^{24}$;

- Experiencing a new episode of acute LBP defined as pain present for more than 24 hours and less than 6 weeks' duration following a period of at least 1 month pain-free ${ }^{24-26}$;

- Did not have known or suspected serious spinal pathology (for example, fracture, malignancy, inflammatory or infective diseases of the spine; cauda equina syndrome or neurological disorder);

- Did not have a history of previous lumbar spinal surgery (eg, spinal fusion, intervertebral disc replacement);

- Did not report suspected or confirmed pregnancy and/or were less than 6 months' post-partum; 
- Did not present with suspected radicular pain (dominant leg pain, positive neural tissue provocation tests and/or any two of altered strength, reflexes or sensation for the same nerve root, assessed clinically);

- Were free from the presence of another painful condition (eg, fibromyalgia, neuropathy, rheumatoid arthritis);

- Did not report serious comorbidities affecting sensorimotor function or causing neurological deficit (eg, multiple sclerosis, spinal cord injury);

- Did not report a history of psychological disorders requiring medication for symptom control (eg, major depressive disorder, bipolar disorder, schizophrenia);

- Demonstrated no contraindications for the application of transcranial magnetic stimulation ${ }^{27}$;

- Provided written informed consent to participate and were able to speak and read English.

\section{Outcome variables: pain and disability}

\section{Primary outcome}

The primary outcome is pain intensity. Self-reported pain scores are determined using the Brief Pain Inventory at T1 and T2. ${ }^{32}$ Participants are asked to score their pain intensity on average over the previous week using an 11-point numerical rating scale (NRS: 0='no pain', $10=$ 'worst pain imaginable'). Pain intensity scores at T2 will also be dichotomised to determine 'recovered' and 'non-recovered' participants. A NRS score of 0 will be classified as recovered LBP and a NRS score $\geq 1$ will be classified as chronic LBP. $^{33}$

\section{Secondary outcome}

The secondary outcome is disability. Self-reported disability will be determined using the 24-point Roland Morris Disability Questionnaire (RMDQ) at T1 and T2. ${ }^{34}$
This questionnaire detects the level of disability experienced as a result of LBP. Disability scores at T2 will be dichotomised with a RMDQ score $\leq 2$ classified as recovered LBP and a RMDQ score $\geq 3$ classified as chronic LBP. $^{33}$

\section{Exposure variables: sensorimotor cortex activity}

Sensory cortex activity in the acute stage of LBP will be assessed using the peak-to-peak area of the $\mathrm{N}_{80}$ and $\mathrm{N}_{150}$ components of the sensory evoked potential (SEP). Motor cortex activity will be assessed using transcranial magnetic stimulation (TMS) derived map volume of the paraspinal muscles at the L3 and L5 spinal level. These procedures have been outlined in detail in the UPWaRD study protocol. ${ }^{31}$ In brief:

SEPs are recorded in response to two blocks of 500 nonnoxious electrical stimuli applied via a constant current stimulator (Digitimer, DS7AH) to the paraspinal muscles $3 \mathrm{~cm}$ lateral to the L3 spinous process, ipsilateral to the side of worst LBP. Electroencephalography (EEG) is recorded using gold plated cup electrodes (Digitimer, Reusable $\mathrm{Au}$ and Ag EEG Cup Electrodes) positioned over $\mathrm{S} 1(3 \mathrm{~cm}$ lateral and $2 \mathrm{~cm}$ posterior to $\mathrm{Cz}$ ) on the side contralateral to worst LBP and referenced to Fz according to the International 10/20 EEG placement system. ${ }^{35}$ The $\mathrm{N}_{80}$ component is thought to represent activity in $\mathrm{S} 1$ (between the first major downward deflection of the curve after stimulation and the first major negative peak, $\mathrm{N}_{80}$ ), the $\mathrm{N}_{150}$ component is thought to represent activity in the secondary sensory cortex (S2) (between the first negative peak, $\mathrm{N}_{80}$, and second negative peak, $\left.\mathrm{N}_{150}\right)$. ${ }^{1011153637}$

L3 and L5 map volume is the measure of total excitability of the corticomotor representation of the paraspinal muscles recorded at L3 and L5 level. $^{38}{ }^{39}$ Participants undergo a standardised TMS

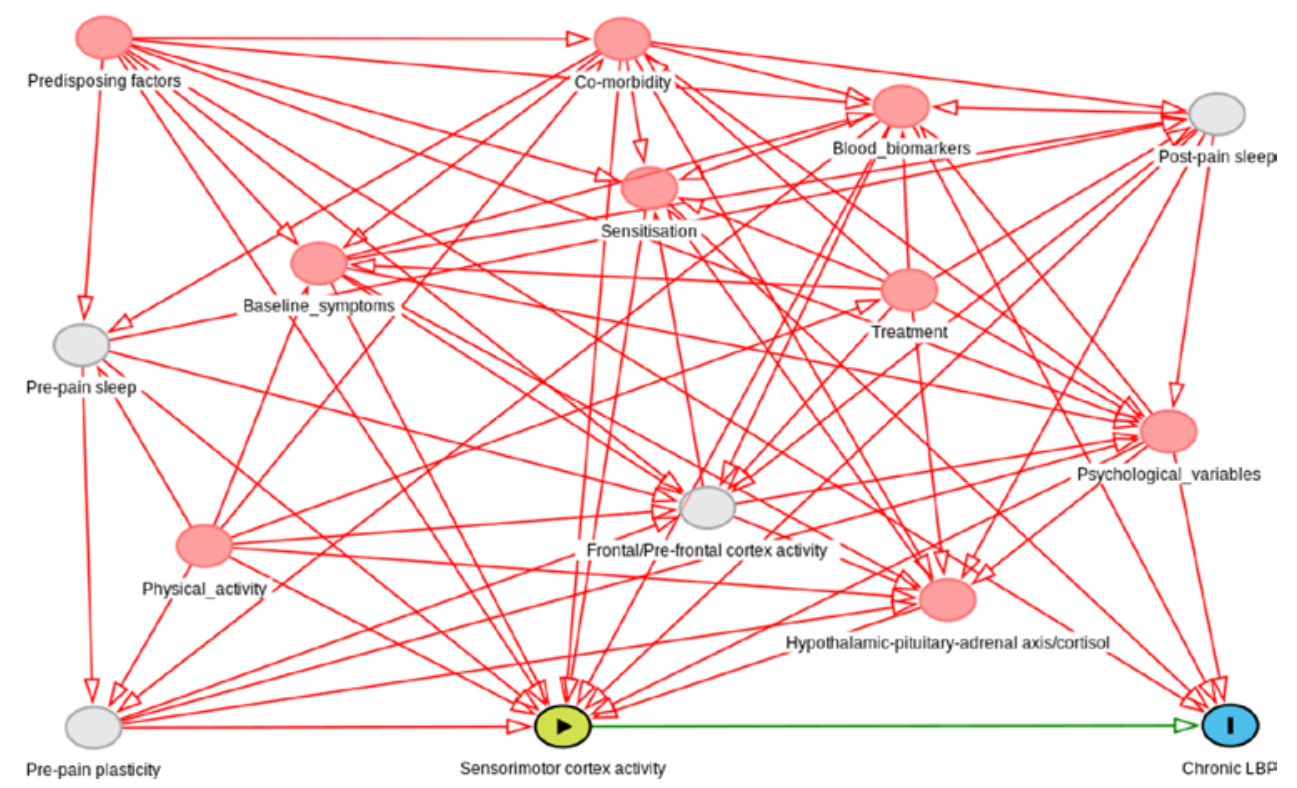

Figure 1 Directed acyclic graph to identify confounders. Confounding variables (theoretical causal effect on exposure (sensorimotor cortex activity) and outcome (chronic LBP)) are in red circles. Grey circles are variables that were unmeasured in the UPWaRD (Understanding persistent Pain Where it ResiDes) study. LBP,low back pain. 
mapping procedure. ${ }^{10}{ }^{40}$ Single-pulse, monophasic TMS is delivered to the M1 contralateral to the side of worst LBP (Magstim 200 stimulator $/ 7 \mathrm{~cm}$ figure-of-eight coil; Magstim Co Ltd, Dyfed, UK). The stimulator intensity is set to $100 \%$, with an interstimulus interval of $\sim 5 \mathrm{~s}$. Surface electromyography (EMG) is recorded from the paraspinal muscles with an electrode (silver-silver chloride disposable electrodes; Noraxon USA Inc, Arizona, USA) placed longitudinally, $3 \mathrm{~cm}$ lateral to the $\mathrm{L} 3$ spinous process and $1 \mathrm{~cm}$ lateral to the $\mathrm{L} 5$ spinous process, ipsilateral to the side of worst LBP. Five stimuli are delivered over pre-marked scalp sites on a $6 \times 7 \mathrm{~cm}$ grid, commencing at the vertex, determined using the International 10/20 System. ${ }^{13} 41$ EMG traces of the five motor evoked potentials recorded at each scalp site are averaged then superimposed over the respective scalp sites to construct a topographical representation of the paraspinal muscle. ${ }^{18}$ All TMS data is analysed using MATLAB 7 (The MathWorks, USA).

\section{Identifying sources of confounding}

A directed acyclic graph was constructed using DAGitty software $^{42}$ to identify all variables that have a plausible, causal effect on the relationship between sensorimotor cortex activity (the exposure) and chronic LBP (the outcome) ${ }^{9}$ Figure 1 details all variables included within the DAG. The DAG outlines explicit assumptions made by the investigators, informed by expert opinion and current literature. $^{19}$

Table 1 details data collected from the UPWaRD study that can be used to control for the identified confounding variables. Procedures for obtaining these variables are outlined in detail in the UPWaRD study protocol. ${ }^{31}$

\section{Limitations}

The DAG methodology is not without limitation, establishing the directionalities of effects in addition to model misspecifications can result in errors, potentially leading to incorrect inferences. ${ }^{19}$ Simpler and more sparse DAGs represent stronger assumptions, as every omission of a variable and its causal pathway represents an assumption of one or more causal null hypotheses. ${ }^{43}$ Further, DAGs do not account for the effect of unmeasured confounding. The effect of unmeasured confounding on the study results will be analysed using a sensitivity analysis. A sensitivity analysis determines how important unmeasured confounding would need to be to alter study conclusions. ${ }^{44}$

Table 1 Confounding variables identified from the directed acyclic graph

\begin{tabular}{|c|c|}
\hline Assessment domain & Confounding variable \\
\hline Predisposing factors & $\begin{array}{l}\text { 1. Age } \\
\text { 2. Sex } \\
\text { 3. Previous history of low back pain: Participants are asked the following question: 'Have you } \\
\text { experienced low back pain in the past?' } \\
\text { 4. BDNF genotype: Cheek swabs taken on the day of baseline testing are used to prepare genomi } \\
\text { DNA (Isohelix DNA Isolation Kit). } \\
\text { 5. Socioeconomic status: Participant postal code is converted into a SEIFA score. } \\
\text { 6. Cultural diversity: Participants are asked the following question: 'How do you define your identit } \\
\text { in ethnic or cultural terms?' }\end{array}$ \\
\hline Blood biomarkers & $\begin{array}{l}\text { 7. BDNF serum concentration: Peripheral venous blood is drawn into serum tubes (BD Vacutainer, } \\
\text { SST II Advance). BDNF serum concentration is measured using an enzyme-linked immunosorbe } \\
\text { assay (ELISA) (Simple Plex Cartridge Kit, Biotrend). } \\
\text { 8. Pro-inflammatory cytokines: Serum samples obtained from the UPWaRD study will also be } \\
\text { analysed for TNF, IL-1 } 1 \beta \text {, IL- } 6 \text { and CRP. Zero is allocated for values below the test sensitivity. }{ }^{53}\end{array}$ \\
\hline
\end{tabular}

Psychological variables 9. PCS: Assesses catastrophising thoughts about pain. The PCS includes 13 items, scored on a 5 -point scale.

10. DASS-21: Includes three 7-item subscales with higher scores indicating greater depression, anxiety and/or stress.

11. PSEQ: Evaluates the confidence of an individual in their ability to perform a range of functional activities while in pain. A total score between 0 and 60 is calculated, higher scores indicate greater self-efficacy beliefs.

Sensitisation

12. Local sensitivity: PPT is measured using a hand-held pressure algometer (Somedic, Hörby, Sweden). The probe (size $1 \mathrm{~cm}^{2}$ ) is applied perpendicular to the skin until the participant reports the sensation has changed from pressure to pain. PPT is measured three times ipsilateral to the side of the worst LBP, $3 \mathrm{~cm}$ lateral to the $\mathrm{L} 3$ spinous process, with the average used for analysis.

13. Distal sensitivity: PPT is measured as above on the thumb nail of the hand ipsilateral to the side of the worst LBP.

BDNF, brain derived neurotrophic factor; CRP, C-reactive protein; DASS-21, Depression, Anxiety and Stress Scale; IL-1 $\beta$, interleukin-1 $\beta$; IL-6, interleukin-6; LBP, low back pain; PCS, Pain Catastrophising Scale; PPT, pressure pain threshold; PSEQ, Pain Self-Efficacy Questionnaire; SEIFA, Socio-economic index for area; TNF, tumour necrosis factor; UPWaRD, Understanding persistent Pain Where it ResiDes. 


\section{Sample size}

G*Power (V.3.0.10, University of Kiel, Germany) was used to calculate the required sample size for estimating the causal effect of baseline sensorimotor activity on chronic LBP. $^{45}$ The minimum sufficient adjustment set identified 16 confounding variables that will be controlled for in the causal model. According to the sample size calculation, 111 participants are required to detect an effect size of 0.2 with $80 \%$ power, using an alpha level of 0.05 , with 16 confounding variables. This calculation is based on detecting a medium effect for a multiple linear regression. $^{46}$

\section{Missing data}

Completeness of data obtained from the UPWaRD study at $\mathrm{T} 1$ and $\mathrm{T} 2$ will be reported as recommended by the STROBE statement. ${ }^{30}$ Cases with missing values will be removed from the data set if follow-up rates are higher than $95 \%$ at T2. If missing data exceeds $5 \%$, multiple imputation will be performed. ${ }^{47}$ The methods used for combining all reported estimates following multiple imputation will be reported (ie, Rubin's rule). ${ }^{47}{ }^{48}$ Where data are missing at random (ie, missing randomly, conditional on covariates), estimates based on multiple imputation are unbiased. ${ }^{49}$

\section{Evaluation of demographics and baseline characteristics}

Data analysis will be performed in $\mathrm{R}$ (The R Foundation for Statistical Computing, a statistical software).$^{50}$ Continuous variables will be presented through centrality measures (mean, median), and dispersion (SD and IQR) according to the distribution, and categorical variables through frequencies and percentages.

\section{Statistical analyses}

The primary outcome, pain intensity, will be entered into a linear regression model as the continuous dependent variable. Separate multivariable linear regression models for the exposure variables $\left(\mathrm{N}_{80}\right.$, SEP component, $\mathrm{N}_{150}$ SEP component, L3 map volume and L5 map volume) will be developed. Confounding variables identified by the DAG will be adjusted for in each linear regression model. Linearity assumptions and model fit will be assessed. ${ }^{451}$ The regression coefficient and corresponding 95\% CIs will be reported and presented in tabular form as recommended by item number 16 a of the STROBE statement. ${ }^{30}$ The probability threshold for statistical significance will be set at $\mathrm{p}<0.05$.

To further explore a possible causal effect of sensorimotor cortex activity during acute LBP on the development of chronic LBP, pain intensity scores at 6 month follow-up will be dichotomised into chronic LBP (NRS score, $\geq 1$ ) or recovered LBP (NRS score, 0$)$. Separate logistic regression models will be created to investigate the causal effect of sensorimotor cortex activity measures and chronic LBP. Adjusted and unadjusted ORs with corresponding CIs will be reported once confounders identified by the DAG are entered into the model. To explore the effect of unmeasured confounding a sensitivity analysis will be performed and reported. ${ }^{44}$

The analysis plan will then be repeated to model the causal effect of sensorimotor cortex activity and the secondary outcome, disability. Any deviations from this protocol will be noted in the final manuscript.

\section{DISCUSSION}

This protocol details an 'a-priori' reported protocol and statistical plan for investigating causal inference using data derived from the UPWaRD prospective cohort study. A directed acyclic graph is presented for the selection of confounding variables, ensuring analytical transparency. Confounding variables entered into a multivariable regression analysis will determine whether sensorimotor cortex activity in the acute stage of LBP has a causal relationship with the development of chronic LBP.

\section{Author affiliations}

${ }^{1}$ School of Science and Health, Western Sydney University, Penrith, New South Wales, Australia

${ }^{2}$ Neuroscience Research Australia (NeuRA), Randwick, New South Wales, Australia ${ }^{3}$ Prince of Wales Clinical School, Faculty of Medicine, University of New South Wales, Sydney, New South Wales, Australia

${ }^{4}$ School of Medical Sciences, Faculty of Medicine, University of New South Wales, Sydney, New South Wales, Australia

\section{Twitter Siobhan M Schabrun @DrSMSchabrun}

Contributors SMS and JHMA acquired funding to undertake this research. LJ, W-JC, VB, CC and ML acquired the original data for this research. SMS, LJ and JHMA formulated the methods and designed the protocol and statistical analysis plan. LJ, SMS and AC drafted the protocol and statistical analysis plan. All authors contributed to revisions and approved the final version of the manuscript.

Funding This work was supported by Grant 1059116 from the National Health and Medical Research Council (NHMRC) of Australia. Funding has been applied to achieve the objectives and outcomes described in this protocol and analysis plan. Funding has been spent only on direct research costs as described within the NHRMC funding agreement. SMS receives salary support from the National Health and Medical Research Council of Australia (1105040).

Competing interests None declared.

Patient consent for publication Not required.

Ethics approval All participants provided written informed consent to participate in the study. Ethical approval was obtained from Western Sydney University Human Research Ethics Committee (H10465) and from Neuroscience Research Australia (SSA: 16/002).

Provenance and peer review Not commissioned; peer reviewed for ethical and funding approval prior to submission.

Open access This is an open access article distributed in accordance with the Creative Commons Attribution Non Commercial (CC BY-NC 4.0) license, which permits others to distribute, remix, adapt, build upon this work non-commercially, and license their derivative works on different terms, provided the original work is properly cited, appropriate credit is given, any changes made indicated, and the use is non-commercial. See: http://creativecommons.org/licenses/by-nc/4.0/.

ORCID iD

Siobhan M Schabrun http://orcid.org/0000-0002-9083-3107

\section{REFERENCES}

1 Vos T, Flaxman AD, Naghavi M, et al. Years lived with disability (YLDs) for 1160 sequelae of 289 diseases and injuries 1990-2010: a systematic analysis for the global burden of disease study 2010. The Lancet 2012;380:2163-96. 
2 Victoria AaO. A problem worth solving, 2013. Available: https://www. msk.org.au/a-problem-worth-solving/

3 Deyo RA, Weinstein JN, Pain LB. Low back pain. N Engl J Med 2001;344:363-70.

4 Costa LdaCM, Maher CG, McAuley JH, et al. Prognosis for patients with chronic low back pain: inception cohort study. $B M J$ 2009;339:b3829.

5 Traeger AC, Lee H, Hübscher M, et al. Effect of intensive patient education vs placebo patient education on outcomes in patients with acute low back pain. JAMA Neurol 2019;76:161.

6 Maher C, Underwood M, Buchbinder R. Non-Specific low back pain. The Lancet 2017;389:736-47.

7 Lee $\mathrm{H}$, Mansell G, McAuley $\mathrm{JH}$, et al. Causal mechanisms in the clinical course and treatment of back pain. Best Pract Res Clin Rheumatol 2016;30:1074-83.

8 Rothman KJ, Greenland S, Lash TL. Modern epidemiology, 2008.

9 Shrier I, Platt RW. Reducing bias through directed acyclic graphs. BMC Med Res Methodol 2008;8:70.

10 Chang W-J, Buscemi V, Liston MB, et al. Sensorimotor cortical activity in acute low back pain: a cross-sectional study. J Pain 2019;20:819-29.

11 Diers M, Koeppe C, Diesch E, et al. Central processing of acute muscle pain in chronic low back pain patients: an EEG mapping study. J Clin Neurophysiol 2007;24:76-83.

12 Schabrun SM, Christensen SW, Mrachacz-Kersting N, et al. Motor cortex reorganization and impaired function in the transition to sustained muscle pain. Cerebral Cortex 2016;26:1878-90.

13 Tsao AH, Danneels WL, Hodges WP. ISSLS Prize winner: Smudging the motor brain in young adults with recurrent low back pain. Spine 2011;36:1721-7.

14 Tsao H, Galea MP, Hodges PW. Reorganization of the motor cortex is associated with postural control deficits in recurrent low back pain. Brain 2008;131:2161-71.

15 Flor $\mathrm{H}$, Braun $\mathrm{C}$, Elbert T, et al. Extensive reorganization of primary somatosensory cortex in chronic back pain patients. Neurosci Lett 1997;224:5-8.

16 Flor H, Elbert T, Knecht S, et al. Phantom-Limb pain as a perceptual correlate of cortical reorganization following arm amputation. Nature 1995;375:482-4.

17 Elgueta-Cancino E, Schabrun S, Hodges P. Is the organization of the primary motor cortex in low back pain related to pain, movement. and/or Sensation? The Clinical journal of pain 2018;34:207-16.

18 Schabrun SM, Elgueta-Cancino EL, Hodges PW. Smudging of the motor cortex is related to the severity of low back pain. Spine 2017:42:1172-8.

19 Lederer DJ, Bell SC, Branson RD, et al. Control of confounding and reporting of results in causal inference studies. guidance for authors from editors of respiratory, sleep, and critical care journals. Ann Am Thorac Soc 2019;16:22-8.

20 Hernán MA. The C-Word: scientific Euphemisms do not improve causal inference from observational data. Am J Public Health 2018;108:616-9.

21 Hernán MA RJ. Causal inference: what if. Boca Raton: Chapman \& Hall/CRC, 2020.

22 Pearl J. An introduction to causal inference. Int J Biostat 2010;6:Article 7.

23 Herbert RD. Cohort studies of aetiology and prognosis: they're different. J Physiother 2014;60:241-4.

24 Greenland S, Morgenstern H. Confounding in health research. Annu Rev Public Health 2001;22:189-212.

25 Hernan MA, Robins JM. Causal inference. FL: CRC Boca Raton, 2010.

26 Greenland S, Pearl J, Robins JM. Causal diagrams for epidemiologic research. Epidemiology 1999;10:37-48.

27 Greenland S. Quantifying biases in causal models: classical confounding vs collider-stratification bias. Epidemiology 2003;14:300-6.

28 Hernán MA, Hernández-Díaz S, Robins JM. A structural approach to selection bias. Epidemiology 2004:15:615-25.

29 Pearl J. Causal inference in statistics: an overview. Stat Surv 2009;3:96-146.
30 Vandenbroucke JP, von Elm E, Altman DG, et al. Strengthening the reporting of observational studies in epidemiology (STROBE): explanation and elaboration. PLoS Med 2007;4:e297.

31 Jenkins LC, Chang W-J, Buscemi V, et al. Do sensorimotor cortex activity, an individual's capacity for neuroplasticity, and psychological features during an episode of acute low back pain predict outcome at 6 months: a protocol for an Australian, multisite prospective, longitudinal cohort study. BMJ Open 2019;9:e029027.

32 Cleeland CS, Ryan K. The brief pain inventory. pain Research Group 1991.

33 Kamper SJ, Maher CG, Herbert RD, et al. How little pain and disability do patients with low back pain have to experience to feel that they have recovered? Eur Spine J 2010;19:1495-501.

34 Roland M, Morris R. A study of the natural history of back pain. Part I: development of a reliable and sensitive measure of disability in lowback pain. Spine 1983;8:141-4.

35 The ten twenty electrode system: International Federation of societies for electroencephalography and clinical neurophysiology. Am J EEG Technol 1961;1:13-19.

36 Bromm B, Lorenz J. Neurophysiological evaluation of pain. Electroencephalogr Clin Neurophysiol 1998;107:227-53.

37 Schabrun SM, Burns E, Hodges PW. New insight into the timecourse of motor and sensory system changes in pain. PLOS One 2015; 10:e0142857.

38 Larivière $C$, Arsenault $A B$, Gravel $D$, et al. Surface electromyography assessment of back muscle intrinsic properties. $J$ Electromyogr Kinesiol 2003;13:305-18.

39 O'Connell NE, Maskill DW, Cossar J, et al. Mapping the cortical representation of the lumbar paravertebral muscles. Clin Neurophysiol 2007;118:2451-5.

40 Schabrun SM, Jones E, Elgueta Cancino EL, et al. Targeting chronic recurrent low back pain from the top-down and the bottom-up: a combined transcranial direct current stimulation and peripheral electrical stimulation intervention. Brain Stimul 2014;7:451-9.

41 Jasper $\mathrm{HH}$. The ten twenty electrode system of the International Federation. Electroencephalogr Clin Neurophysiol Suppl 1958:10:371-5

42 Textor J, van der Zander B, Gilthorpe MS, et al. Robust causal inference using directed acyclic graphs: the R package 'dagitty'. International Journal of Epidemiology 2016;45:1887-94.

43 Greenland S. For and against methodologies: some perspectives on recent causal and statistical inference debates. Eur J Epidemiol 2017;32:3-20.

44 VanderWeele TJ, Ding P. Sensitivity analysis in observational research: introducing the E-value. Ann Intern Med 2017;167:268-74

45 Faul F, Erdfelder E, Lang A-G, et al. G*Power 3: a flexible statistical power analysis program for the social, behavioral, and biomedical sciences. Behav Res Methods 2007;39:175-91.

46 Ferguson CJ. An effect size primer: a guide for clinicians and researchers. Prof Psychol 2009;40:532-8.

47 Moons KGM, Altman DG, Reitsma JB, et al. Transparent reporting of a multivariable prediction model for individual prognosis or diagnosis (TRIPOD): explanation and elaboration. Ann Intern Med 2015;162:W1-73

48 Marshall A, Altman DG, Holder RL, et al. Combining estimates of interest in prognostic modelling studies after multiple imputation: current practice and guidelines. BMC Med Res Methodol 2009;9:57.

49 Kenward MG, Carpenter J. Multiple imputation: current perspectives. Stat Methods Med Res 2007;16:199-218.

50 Core Team R. R: a language and environment for statistical computing. Vienna: R Foundation for statistical computing, 2013.

51 Hulley SB. Designing clinical research. Lippincott Williams \& Wilkins, 2007.

52 Pink B. Socio-Economic indexes for areas (SEIFA. Canberra: Australian Bureau of Statistics, 2011.

53 Klyne DM, Barbe MF, van den Hoorn W, et al. ISSLS Prize in clinical science 2018: longitudinal analysis of inflammatory, psychological, and sleep-related factors following an acute low back pain episode -the good, the bad, and the ugly. Eur Spine $J$ 2018;27:763-77. 